\title{
Dose-dependent requirement of patched homologue 1 in mouse pancreatic beta cell mass
}

\author{
S. Nakayama • M. Arakawa • T. Uchida • T. Ogihara • \\ R. Kanno • F. Ikeda • K. Azuma • T. Hirose • \\ R. Kawamori $\cdot$ Y. Fujitani $\cdot$ H. Watada
}

Received: 3 March 2008 / Accepted: 23 May 2008 / Published online: 25 July 2008

(C) Springer-Verlag 2008

\begin{abstract}
Aims/hypothesis Ectopic activation of hedgehog (HH) signalling in pancreas induces various abnormal morphogenetic events in the pancreas. This study analysed the dose-dependent requirement of patched homologue 1 (PTCH1), a negative regulator of $\mathrm{HH}$ signalling on pancreatic development.

Methods We used a recessive spontaneous mutant mouse denoted as mes which carries a mutated Ptch1 resulting in deletion of the most carboxy-terminal cytoplasmic domain of the PTCH1 protein. In this study, we analysed pancreatic morphology in $P t c h 1^{+/+}$, Ptch $^{+/ \text {mes }}$, Ptch $^{+/-}$, Ptch $^{\text {mes/mes }}$ and $P t c h 1^{-/ m e s}$ mouse embryos, as well as the islet mass in adult Ptchl $^{+/+}$, Ptch $^{+/ \text {mes }}$ and Ptch $1^{+-}$mice.

Results Until embryonic day (E) 12.5, no obvious abnormality of pancreas was observed in any of the Ptchl mutants. The levels of PDX1 and glucagon were also not evidently different among the mice genotypes studied. Thereafter, morphological abnormalities appeared in the Ptchl mutant mice. The beta, alpha and exocrine cell masses decreased at E18.5 in parallel with increased HH signalling, with beta cell
\end{abstract}

Electronic supplementary material The online version of this article (doi:10.1007/s00125-008-1080-2) contains supplementary material, which is available to authorised users.

S. Nakayama $\cdot$ M. Arakawa $\cdot$ T. Uchida $\cdot$ T. Ogihara $\cdot$ R. Kanno $\cdot$

F. Ikeda $\cdot$ K. Azuma $\cdot$ T. Hirose $\cdot$ R. Kawamori $\cdot$ Y. Fujitani $\cdot$

H. Watada $(\triangle)$

Department of Medicine, Metabolism and Endocrinology,

Juntendo University School of Medicine,

2-1-1 Hongo, Bunkyo-ku,

Tokyo 113-8421, Japan

e-mail: hwatada@med.juntendo.ac.jp

T. Hirose $\cdot$ R. Kawamori $\cdot$ Y. Fujitani

Center for Therapeutic Innovations in Diabetes,

Tokyo, Japan mass showing the highest sensitivity to $\mathrm{HH}$ signalling with a significant decrease even in $P t c h 1^{+/ m e s}$ mice. Adult Ptch $1^{+/-}$ mice also showed a significant decrease in beta cell mass compared with wild-type mice.

Conclusions/interpretation Our findings indicate that the carboxy-terminal domain of Ptch1 is essential for pancreatic development. In addition, the loss of Ptchl function decreases both the endocrine and exocrine cell mass in a dose-dependent manner, with beta cells particularly sensitive to changes in $\mathrm{HH}$ signalling.

Keywords Hedgehog · Insulin · Islets · Patched 1 . Ptc1 Ptch 1 Type 2 diabetes

\begin{tabular}{|c|c|}
\hline Abbreviatio & \\
\hline alpha-SMA & alpha-smooth muscle actin \\
\hline $\mathrm{E}$ & embryonic day \\
\hline EMT & epithelial to mesenchymal transition \\
\hline $\mathrm{HH}$ & hedgehog \\
\hline IPGTT & intraperitoneal glucose tolerance test \\
\hline PTCH1 & patched homologue 1 \\
\hline SHH & sonic hedgehog \\
\hline
\end{tabular}

Introduction

Hedgehog $(\mathrm{HH})$ signalling regulates morphogenesis and cell differentiation in various tissues [1]. There are at least three secreted mammalian $\mathrm{HH}$ ligands, including sonic hedgehog (SHH), Indian hedgehog (IHH) and desert hedgehog (DHH) [1]. While their binding affinities differ slightly, all $\mathrm{HH}$ proteins bind to transmembrane receptors, patched homologue 1 (PTCH1) and 2 (PTCH2) in target cells. The expression of PTCH1 is more prominent and its function has been analysed in detail [2]. In the absence of 
ligand, PTCH1 represses the activity of Smo, a G-protein coupled-like receptor. Upon ligand binding, repression of Smo activation is alleviated, allowing nuclear translocation of Gli transcription factors and subsequent expression of HH target genes including Gli itself [3].

During early endoderm differentiation, both $S h h$ and $I h h$ are expressed strongly throughout the endodermal epithelium, with the exception of pancreatic primordia [4-6]. Similarly, Ptchl is expressed in the mesenchyme adjacent to the pancreas, but expression has not been confirmed in the pancreas area of mouse embryos before embryonic day (E) 12.5 [4]. Thereafter, the developing tissue starts to express several HH components $[7,8]$. The expression of Ptch1 has been confirmed by RT-PCR assay after E13.5, and mature pancreas expresses Ihh, Dhh, Hhip, Ptchl and Smo in islet and ductal cells [8-11]. Several groups have investigated the effect of increased $\mathrm{HH}$ signalling on pancreatic morphogenesis. Ectopic expression of $\mathrm{SHH}$ under the control of the $P d x 1$ promoter results in almost complete organ ablation [4, 12]. In addition, ectopic expression of both SHH and IHH under the control of the Pax4 promoter induces a decreased pancreatic mass [12]. These findings suggest that the limited HH signalling in the pancreas plays a key role in pancreatic organogenesis.

A previous study suggested that $\mathrm{HH}$ signalling affects pancreatic development dose-dependently [10]. Loss of the HH pathway inhibitor, Hhip, increased the formation of pancreatic mesenchyme and decreased the size of the pancreatic epithelium. The additional loss of one Ptchl allele in Hhip mutant mice caused a more profound loss of epithelial cells, indicating that pancreatic cells respond to different levels of HH activity [10]. However, the dosedependency of $\mathrm{HH}$ activity in later-stage pancreatic development could not be fully analysed using these models because loss of one Ptch1 allele in the Hhip mutant was lethal before E13.

Previous studies have revealed that the carboxy-terminal cytoplasmic domain of Patched plays an essential role in blocking $\mathrm{HH}$ signalling in wing disc [13], as well as regulating Patched degradation and trafficking [14] in Drosophila. mes encodes a mutant PTCH1 protein lacking most of the carboxy-terminal 220 amino acids [13] and is considered a hypomorphic allele. Organ development phenotypes in compound heterozygotes of mes and Ptch1 null alleles $\left(P t c h 1^{\text {mes/ }}\right)$ are generally more severe than those for mes homozygotes (Ptch1 ${ }^{\text {mes/mes }}$ ), but milder than those for homozygotes of Ptch1 null alleles $\left(P t c h 1^{-/}\right)$[13]. The Ptch $1^{\text {mes/- }}$ mice survive longer than Ptch1 null homozygotes, making them a useful tool to study the biological roles of PTCH1 in later development. Interestingly, although mes is considered to be a hypomorphic allele, Ptch $1^{\text {mes } /-}$ mice show no patterning defect of the developing spinal cord where titration of $\mathrm{HH}$ activation plays an essential role $[13,15,16]$. Thus, the carboxy-terminal domain of PTCH1 has a qualitatively distinct function depending on tissue or developmental stage $[15,17]$.

Here, we used mes mice to investigate the role of the PTCH1 carboxy-terminal domain in pancreatic development and the dose-dependent effects of increased $\mathrm{HH}$ signalling. We generated $P t c h 1^{+/+}, P t c h 1^{+/ m e s}, P t c h 1^{+/-}$, Ptch $1^{\text {mes/mes }}$ and $P t c h 1^{-/ m e s}$ mice to analyse pancreatic morphology during development in each mouse. Our data implicated the carboxy-terminal domain of PTCH1 in pancreatic development.

\section{Methods}

Animals The Animal Care and Use Committee of Juntendo University, School of Medicine reviewed and approved the study protocol. All mice were housed in specific pathogenfree barrier facilities, maintained in a $12 \mathrm{~h}$ light-dark cycle and had free access to standard rodent food (Oriental Yeast, Osaka, Japan) and water. Ptch1 knockout mice, ROSA26lacZ mice and Ptfla-Cre mice were generated as described previously [18-20]. Ptchl $1^{+/ \text {mes }}$ mice were obtained from Jackson Laboratories (Bar Harbor, ME, USA). Ptch1 mutant mice used to investigate embryonic development were maintained in a CD1 background. To analyse the adult phenotype, the Ptch1 mutant mice were backcrossed with C57BL/6J mice for a minimum of ten generations. Mutant mice and embryos were genotyped as described previously $[15,18-20]$.

$R N A$ preparation and RT-PCR analysis Dissected E14.5 pancreas was lysed in TRIzol reagent (Invitrogen, Carlsbad, CA, USA) and first-strand cDNA was synthesised using $1 \mu \mathrm{g}$ of total RNA with oligo dT primers and superscript reverse transcriptase (Invitrogen). For quantitative QuantiProbePCR (Qiagen, Hilden, Germany), cDNA was analysed using an ABI 7700 PCR machine (Applied Biosystems Japan, Tokyo, Japan) with $2 \times$ QuantiTect Probe PCR Master Mix (Qiagen) and specific probes. The thermal cycle for amplification was as follows: $2 \mathrm{~min}$ at $50^{\circ} \mathrm{C}$, $15 \mathrm{~min}$ at $95^{\circ} \mathrm{C}$ and 40 cycles of $94^{\circ} \mathrm{C}$ for $15 \mathrm{~s}, 56^{\circ} \mathrm{C}$ for $30 \mathrm{~s}$ and $76^{\circ} \mathrm{C}$ for $30 \mathrm{~s}$. The relative mRNA expression levels were calculated by the cycle threshold method with standard cDNA. The threshold is manually adjusted to a value above the background and significantly below the plateau of an amplification plot. The cycle at which amplification plot crosses the threshold is determined as threshold cycle. Quantification of target amounts was calculated by comparative cycle threshold method [21]. $\beta$ Actin mRNA served as an internal invariant standard. The primers and probes used are shown in Electronic supplementary material (ESM) Table 1. 
Table 1 Relative Gli1 mRNA expression and body weight in each Ptch1 mutant

\begin{tabular}{|c|c|c|c|c|c|}
\hline & $\operatorname{Ptchl}^{+/+}$ & Ptch1 $1^{+/ m e s}$ & $\operatorname{Ptch1}^{+/-}$ & Ptchl $1^{\text {mes/mes }}$ & Ptch1 $1^{-/ m e s}$ \\
\hline Gli1 & $1.0 \pm 0.2$ & $2.8 \pm 0.4$ & $4.7 \pm 1.6$ & $77.8 \pm 26.5$ & $294 \pm 168$ \\
\hline Body weight (g) & $1.51 \pm 0.04$ & $1.49 \pm 0.03$ & $1.56 \pm 0.07$ & $1.68 \pm 0.06$ & $1.95 \pm 0.04$ \\
\hline
\end{tabular}

Data are mean \pm SEM. Gli1 mRNA expression levels were measured by real-time RT-PCR analysis using E14.5 pancreas of each mice $(n=6$ each). Values are shown relative to the level of $\beta$-actin mRNA. To facilitate comparison, the expression in wild-type pancreas was adjusted to 1 . Body weight was measured at E18.5 ( $n=5$ each)

Immunohistochemical analysis Isolated pancreas or pancreas-containing tissues were fixed for $4 \mathrm{~h}$ at $4^{\circ} \mathrm{C}$ in $4 \%$ paraformaldehyde in phosphate-buffered saline (wt/wt). The fixed tissue was embedded in paraffin and then cut into $5 \mu \mathrm{m}$ thick sections and mounted on microscope slides. Immunohistochemical analysis was carried out as described previously [22] using the following primary antibodies at the indicated dilutions: mouse anti-vimentin antibody (1:200; Sigma-Aldrich Japan, Tokyo, Japan); rabbit antiPDX1 antibody [23], 1:5,000; guinea pig anti-insulin antibody (1:1,000; Linco Research, St Charles, MO, USA); rabbit anti-glucagon antibody (1:1,000; Dako, Carpentaria, CA, USA); rabbit anti-amylase antibody (1:2,500; Sigma-Aldrich); mouse anti-somatostatin antibody $(1: 1,000$; Biogenesis, Sandown, NH, USA); rat antipancreatic polypeptide antibody (1:500; Linco Research); rabbit anti-musculoaponeurotic fibrosarcoma oncogene family, protein A (MAFA) antibody (1:2,500; Bethyl Laboratories, Montgomery, TX, USA); mouse anti-Ecadherin antibody (1:50; Santa Cruz Biotechnology, Santa Cruz, CA, USA); and rat anti-bromodeoxyuridine antibody (1:50; OBT, Oxford, UK). Secondary antibodies were as follows: biotinylated goat anti-rabbit IgG (Vector Laboratories, Burlingame, CA, USA), biotinylated goat anti-rat IgG (Cedarlane, Hornby, ON, Canada), biotinylated goat anti guinea pig IgG (Chemicon International, Temecula, CA, USA), goat anti-mouse IgG conjugated with Alexa 488 (Molecular Probes, Eugene, OR, USA) and goat antirabbit IgG conjugated with Alexa 568 (Molecular Probes). To identify proliferating cells in the E14.5 pancreas, BrdU was injected and viewed as described previously [24]. With regard to all antibodies, we performed immunostaining without first or secondary antibodies and confirmed that the immunostaining shown in this study was not artefactual.

The positively-stained cell area was determined as a percentage on insulin-, glucagon- and amylase-stained sections viewed on a bright-field microscope (E800; Nikon, Tokyo, Japan) connected to an XYZ controller and digital camera (Sony, Tokyo, Japan). Each area was determined on four to six immunostained sections of three to seven mice each, with each section separated by at least $100 \mu \mathrm{m}$. The cell areas were analysed using image analysis software
(Image Pro4.5J; Planetron, Tokyo, Japan). Non-specific staining was excluded from the quantification. The percentage of the cell area was calculated by the following formula: cell area $(\%)=$ the area stained by antibody/the total pancreatic area. Glucagon-positive area/exocrine area and insulin-positive area/exocrine area were calculated by the area stained by glucagon or insulin divided by exocrine area judged by haematoxylin staining.

$X$-gal staining $\mathrm{X}$-gal staining was carried out as described previously [25].

Glucose and insulin tolerance tests Intraperitoneal glucose tolerance test (IPGTT) was carried out on 12-week-old male $P t c h 1^{+/+}, P t c h 1^{+/ m e s}$ and Ptch $1^{+/-}$mice with $2.0 \mathrm{~g} / \mathrm{kg}$ glucose. The insulin tolerance test was carried out with 0.75 units $/ \mathrm{kg}$ insulin as described previously [26].

Data analysis Results are presented as mean \pm SEM. Differences between two groups were examined for statistical significance using the unpaired Student's $t$ test. Statistical differences among three or more groups were examined by one-way ANOVA followed by Scheffé's post hoc test. A $p$ value $<0.05$ was considered significant.

\section{Results}

Role of carboxy-terminal domain of PTCH1 in pancreatic development To investigate the involvement of carboxyterminal domain of PTCH1 in the development of pancreas, we generated Ptch1 ${ }^{+/+}$, Ptch $1^{+/ m e s}, P t c h 1^{+/-}$, Ptch $1^{\text {mes/mes }}$ and Ptch $1^{-/ m e s}$ mice. We quantified HH signalling in the developing pancreas from the expression of $\mathrm{HH}$ target gene Gli1 mRNA, using total RNA isolated from E14.5 pancreas. The presence of mes alleles, together with loss of wild-type Ptch1 alleles, led to a stepwise increase in $\mathrm{HH}$ signalling in the pancreas (Table 1). These results clearly demonstrate that deletion of the carboxy-terminal domain of PTCH1 increases $\mathrm{HH}$ signalling during pancreatic development. In parallel with the increased $\mathrm{HH}$ signalling, we noted a dose-dependent increase in body weight at E18.5 (Table 1), consistent with previous findings [15]. 
The effect of dysfunctional PTCH1 on early stage of pancreatic differentiation Macroscopic analysis of E10.5 and E12.5 pancreas from each Ptch1 mutant revealed no obvious reduction in pancreas organ size (data not shown). In the early stage of pancreatic differentiation, all pancreatic epithelial cells express PDX1 [27], which is thus considered to be a good marker of pancreatic epithelial cells. We compared the expression of PDX1 in E10.5 and E12.5 pancreas from each Ptch1 mutant. However, no obvious differences were observed (Fig. 1a). Next, we investigated the differentiation of endocrine cells in E10.5 and E12.5 pancreas. In E10.5 pancreas, we easily detected glucagonpositive cells in each Ptch1 mutant and did not find any obvious differences in the number of glucagon-positive cells in E10.5 and E12.5 pancreas (Fig. 1b). On the other hand, in E10.5 pancreas, we hardly detected any insulinpositive cells in any of the Ptch1 mutants (data not shown). In E12.5 pancreas, we were able to detect insulin-positive cells to a similar degree to that seen in each Ptch1 mutant (data not shown). These results suggest that the dysfunction of Ptch1 does not result in obvious changes in cell number of epithelial cells and in the differentiation into endocrine cells in pancreas until E12.5.

The effect of dysfunctional PTCH1 on exocrine pancreas at late stage of pancreatic differentiation Macroscopic analysis of E15.5 and E18.5 pancreas from each Ptch1 mutant revealed an obvious reduction in pancreas organ size in Ptch ${ }^{\text {mes/mes }}$ mice, while the size of the surrounding duodenum was comparable among the Ptchl mutants. The effect was more remarkable in $P t c h 1^{-/ m e s}$ mice (Fig. 2a). Vimentin and amylase double-staining of E18.5 pancreas revealed an increase in the mesenchyme and a concurrent decrease in acinar cell area in Ptchl ${ }^{\text {mes/mes }}$ mice and Ptchl ${ }^{-/ m e s}$ mice, the result being more striking in the latter (Fig. 2b).

To quantitatively assess the effect of dysfunctional Ptch1 on the number of acinar cells in the pancreas, we investigated the levels of amylase in E15.5 and E18.5 pancreas (ESM Fig. 1). As early as E15.5, a decreased number of pancreatic acinar cells in Ptchl mutant was
Fig. 1 The effect of dysfunctional PTCH1 on early stages of pancreatic differentiation. a PDX1 immunostaining of pancreas at E10.5 and E12.5 in mice as indicated. Scale bar, $50 \mu \mathrm{m}$. b Glucagon staining of pancreas at E10.5 and E12.5. Scale bar, $50 \mu \mathrm{m}$
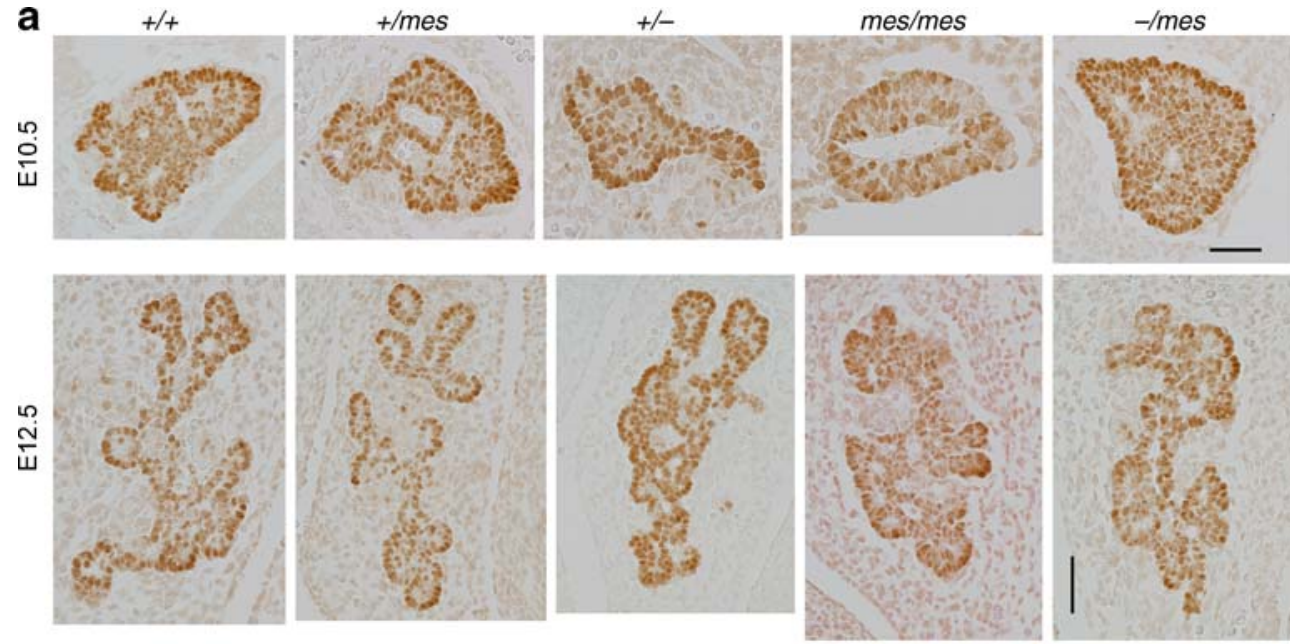

b
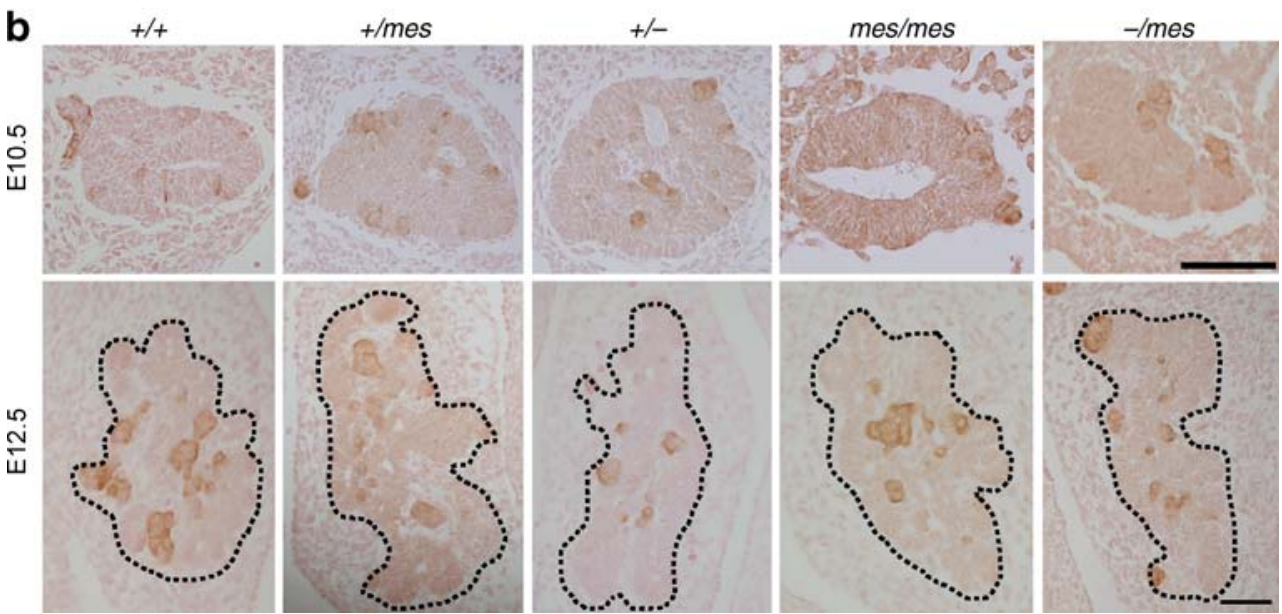
Fig. 2 Exocrine cell mass in Ptch1 mutants. a Macroscopic analysis of E15.5 and E18.5 pancreas from $P t c h 1^{+/+}$, Ptchl $^{+/ m e s}$, Ptchl $^{+-}$, Ptch $^{\text {mes } m e s}$ and $P t c h 1^{-/ m e s}$ mice. The black dotted line marks the pancreas. dp, dorsal pancreas; sp, spleen; $\mathrm{vp}$, ventral pancreas. b Vimentin (green) and amylase (red) staining of E18.5 pancreas from each Ptch1 mutant as indicated. c Percentages of amylase-immunopositive areas in E18.5 pancreas from each Ptchl mutant. To facilitate comparison, amylaseimmunopositive areas in wildtype pancreas quantifications were adjusted to 1 . Data are mean \pm SEM. Ptch $1^{+/ 4}, n=8$; PtchI $^{+/ \text {mes }}, n=8 ;$ Ptchl $^{+/}, n=7$; Ptch1 ${ }^{\text {mes/mes }}, n=5 ;$ Ptch $1^{- \text {mes }}$, $n=6 ;{ }^{*} p<0.05$ vs $P$ tchl ${ }^{+/+}$; $\dagger p<0.05$ vs $P$ tch $1^{+/ m e s}$; $\$ p<0.05 \mathrm{vs} \mathrm{Ptch}^{+/}$ a
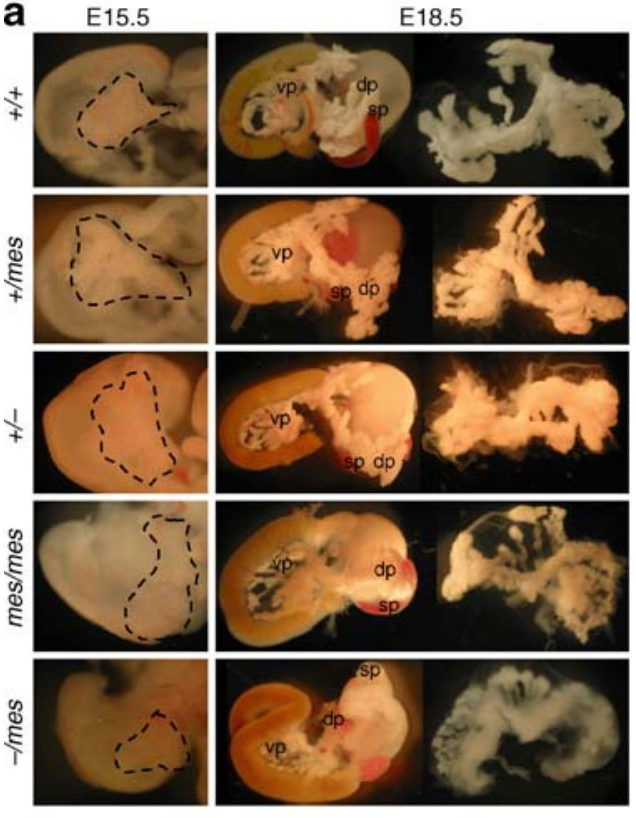

C

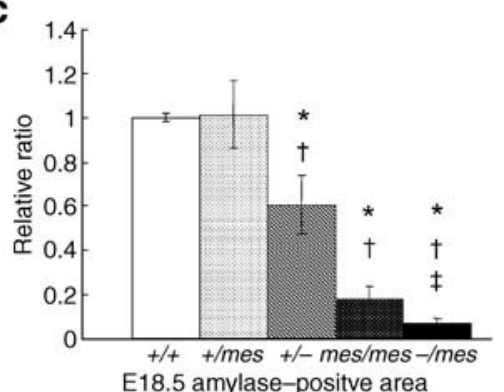

E18.5 amylase-positve area b

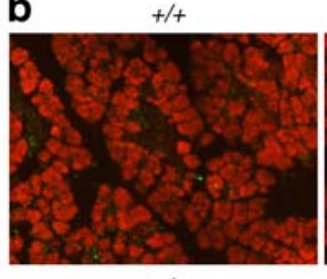

$+/$
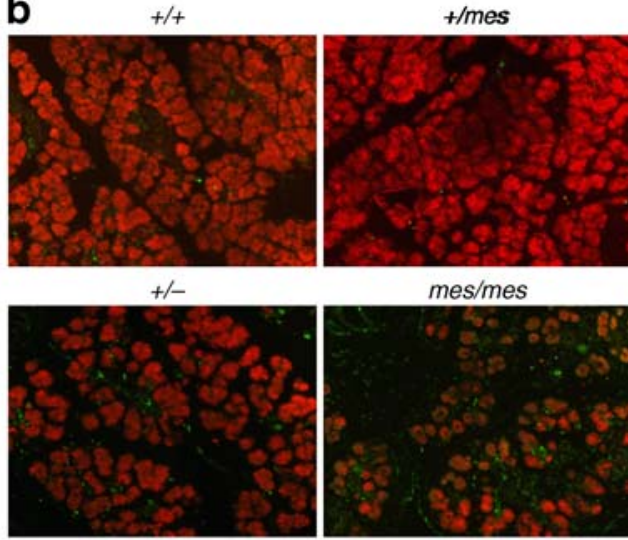

mes/mes

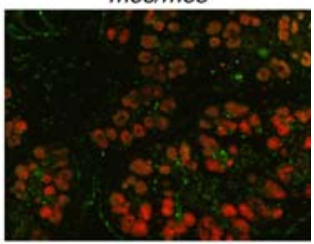

$-/ m e s$

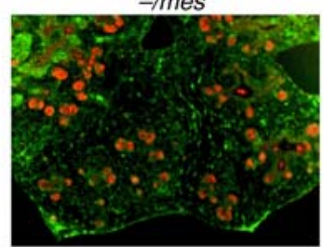

observed. Quantitative analysis in E18.5 pancreas confirmed a marked decrease in amylase-positive cell area in Ptch $1^{\text {mes/mes }}$ and Ptch $1^{-/ \text {mes }}$ mice, as well as in $P t c h 1^{+-}$mice (Fig. 2c). These results indicate that the exocrine cell mass decreases in parallel with an increase in $\mathrm{HH}$ signalling.

The effect of dysfunctional PTCH1 on endocrine pancreas at late stage of pancreatic differentiation Next, we investigated the endocrine pancreatic cell area at E15.5 and E18.5. At E15.5, the glucagon-positive cell area was decreased in pancreas of Ptch1 mutant mice (Fig. 3a). Quantitative analysis of E18.5 pancreas revealed a correlation between the decrease in glucagon-positive cell area and the increase in $\mathrm{HH}$ signalling (Fig. 3b). To investigate whether dysfunctional PTCH1 specifically altered glucagon-positive area, we calculated glucagon-positive area: exocrine area ratio in pancreas of wild-type, Ptch $1^{+/ m e s}$ and Ptch $^{+/-}$mice. As shown in Fig. 3c, there were no differences in the glucagon-positive area:exocrine cell area ratio among any of the mice. These results suggest that dysfunctional PTCH1 results in a proportional decrease of glucagonpositive area with a decrease of exocrine cell area.
At E15.5, the insulin-positive cell area was also decreased in pancreas of Ptchl mutant mice (Fig. 4a). Quantitative analysis of E18.5 pancreas revealed a correlation between the decrease in insulin-positive cell area and the increase in $\mathrm{HH}$ signalling. The reduction of insulinpositive cells even in $P t c h 1^{+/ m e s}$ mice suggested that the beta cell mass was particularly sensitive to changes in $\mathrm{HH}$ signalling (Fig. 4b). To investigate whether dysfunctional Ptch1 specifically altered the insulin-positive area, we calculated the insulin-positive area:exocrine cell area ratio in pancreas of wild-type, Ptch $1^{+/ m e s}$ and $P t c h 1^{+/-}$mice. As shown in Fig. 4c, the insulin-positive area/exocrine cell area ratio in pancreas was associated with increased $\mathrm{HH}$ signalling. These results suggest that the subtle dysfunction of PTCH1 results in a specific decrease of insulin-positive area in pancreas.

Immunostaining of somatostatin and pancreatic polypeptide in the pancreas of each Ptch1 mutant at E18.5 revealed that delta and pancreatic polypeptide cells also tended to decrease according to the increase in the $\mathrm{HH}$ signal, although quantitative analysis was not performed (data not shown). Thus, increased HH signalling induces a decrease 
Fig. 3 Alpha cell mass in each Ptch1 mutant. a Glucagon staining of pancreas at E15.5 and E18.5 in mutants as indicated. Scale bar, $50 \mu \mathrm{m}$. b Percentages of glucagon-immunopositive areas in pancreas of each Ptch1 mutant at E18.5. To facilitate comparison, glucagonimmunopositive area in wildtype pancreas was adjusted to 1 . Data are mean \pm SEM. Ptch $1^{+/+}$, $n=7 ;$ Ptchl $^{+/ m e s}, n=6 ;$ Ptch $^{+/}$, $n=7$; Ptch $1^{\text {mes } / \text { mes }}, n=5$;

Ptch $1^{-/ \text {mes }}, n=3 ; * p<0.05$ vs Ptch $^{+/+} ; \dagger p<0.05$ vs Ptch $1^{+/ m e s}$ $\ddagger p<0.05$ vs Ptch $^{+/} ; \S p<0.05$ vs Ptchl ${ }^{\text {mes/mes }}$. c Glucagonpositive area:exocrine cell area ratio in pancreas of each Ptch1 mutant at E18.5. Data are mean \pm SEM, $n=7$
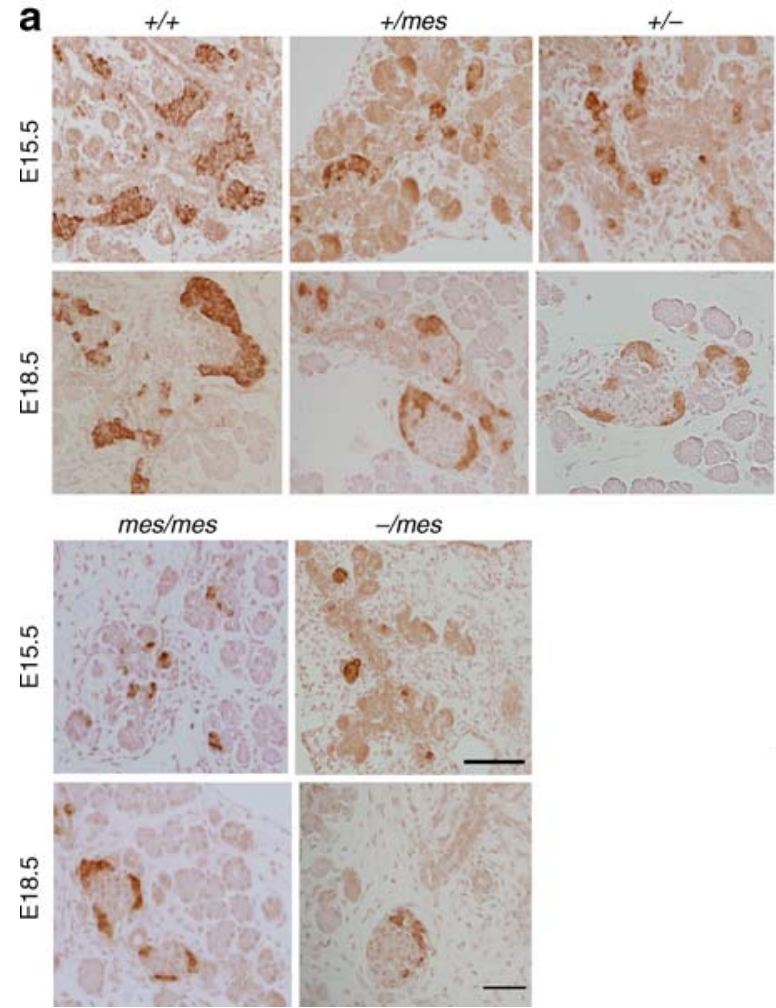

b
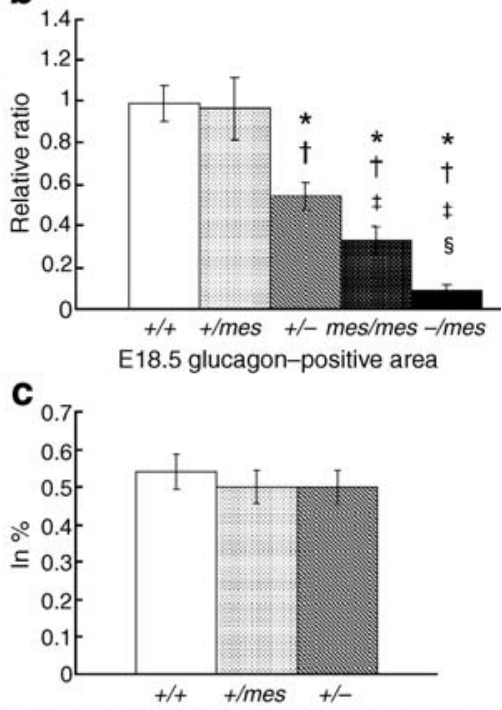

Glucagon-positive area:exocrine pancreas area in the mass of all four endocrine pancreas cell types in a dose-dependent manner. Among them, the number of insulin-positive cells seemed to be specifically decreased in the Ptch1 mutants.
Effect of dysfunctional PTCH1 on transcription factors implicated in beta cell differentiation Ngn3 (also known as Neurog3) is a presumed marker of endocrine precursor cells $[28,29]$. We next investigated whether the differentiation
Fig. 4 Beta cell mass in each Ptch1 mutant. a Insulin staining of pancreas at E15.5 and E18.5 in mutant mice as indicated. Arrowheads indicate insulinpositive cells. Scale bar, $50 \mu \mathrm{m}$. b Percentages of insulin-immunopositive areas in pancreas of each Ptch1 mutant at E18.5. To facilitate comparison, insulinimmunopositive area in wildtype pancreas has been adjusted to 1 . Data are mean $\pm \mathrm{SEM}$. Ptch $1^{+/+}, n=7 ;$ Ptch $^{+/ m e s}, n=6$; Ptchl $^{+-}, n=7 ;$ Ptchl ${ }^{\text {mes/mes }}$, $n=5 ;$ Ptch $^{-/ m e s}, n=3 ;{ }^{*} p<0.05$ vs $P$ tch $1^{+/+} ; \dagger p<0.05$ vs Ptch $1^{+/ \text {mes }} ; \ddagger p<0.05$ vs Ptch $^{+/}$; $\S p<0.05$ vs $P$ tch $1^{\text {mes/mes }}$.

c Insulin-positive area:exocrine cell area ratio in pancreas of each Ptch1 mutant at E18.5. Data are mean \pm SEM $(n=7$ each $) ;{ }^{*} p<0.05$ vs Ptchl $^{+/+}$
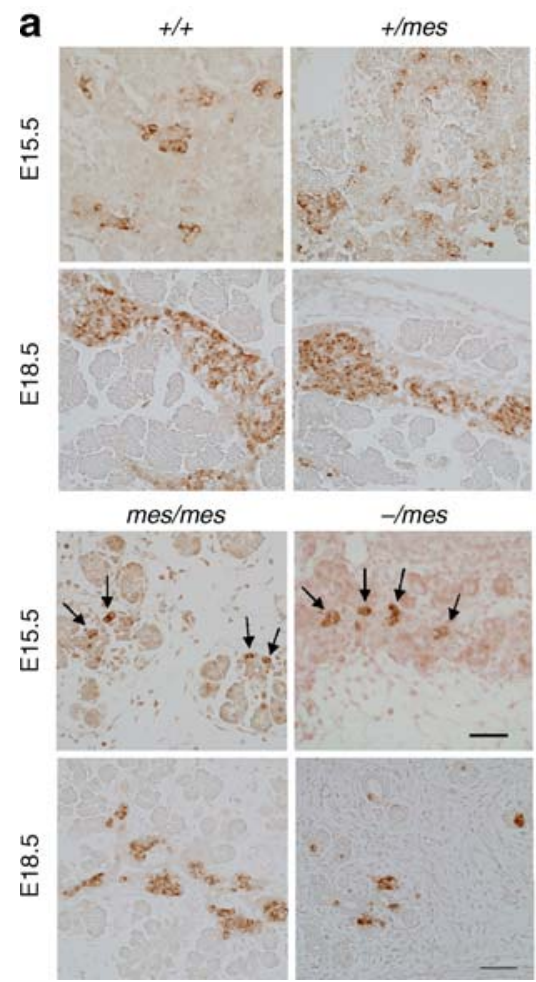

b
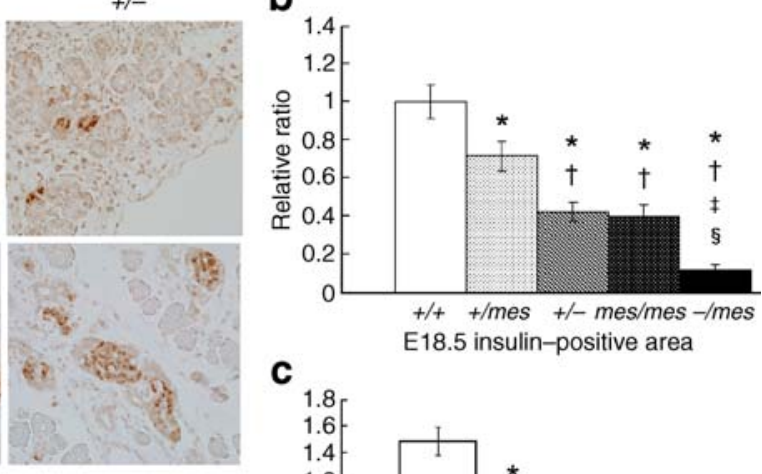

C

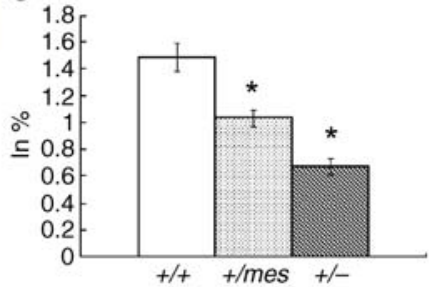

Insulin-positive area:exocrine pancreas area 
from immature pancreatic epithelial cells to Ngn3-expressing endocrine precursor cells is affected by increased $\mathrm{HH}$ signalling. Quantification of Ngn3 expression using RNA isolated from E14.5 pancreas revealed a decrease in parallel with the increased $\mathrm{HH}$ signalling (Fig. 5a). However, the reduction in Ngn3 expression was relatively modest compared with the reduction in alpha and beta cell mass. Thus, these results suggest that increased $\mathrm{HH}$ signalling could induce both a decreased fate commitment into endocrine cells and a reduced expansion of endocrine cells. In the late stage of pancreas differentiation, the expression of PDX1 is gradually restricted to pancreatic beta and delta cells. Reflecting the decreased insulin-positive cell number, PDX1-positive cells decreased in Ptch $1^{\text {mes/mes }}$ and Ptch1 ${ }^{-/ m e s}$ mice at E15.5 and E18.5 (ESM Fig. 2). In addition, the expression of MAFA at E18.5, a marker of fully mature beta cells [30], also decreased in parallel with the increased $\mathrm{HH}$ signalling (Fig. 5b).

Increased mesenchyme induced by dysfunctional PTCH1 is not derived from pancreatic epithelial cells The observed increase in mesenchymal cells and concurrent decrease in epithelial cells in the pancreas is a well-known phenotype of mice with increased $\mathrm{HH}$ signalling in pancreas [4, 10, 11]. Previous studies have suggested that $\mathrm{HH}$ signalling attenuates fibroblast growth factor 10 signalling, which regulates the proliferation of pancreatic epithelial cells [11, 31]. Thus, increased $\mathrm{HH}$ signalling results in a reduction of the pancreatic epithelial cells. On the other hand, no studies have investigated the possibility that the increased mesenchyme is formed by an epithelial to mesenchymal transition (EMT) in pancreas of Ptch1 mutants [32-34]. To investigate whether the increased mesenchymal cells are indeed the result of an EMT, we performed Cre-lox recombination studies based on in vivo genetic lineage tracing. Pancreas specific transcription factor, 1a (PTF1A) is expressed on all epithelial cells. In the pancreas, it localises in pancreatic buds, but not in neighbouring epithelial or mesenchymal cells. We crossed Ptfla-Cre mice with ROSA26-lacZ mice, which carry a modified lacZ gene driven by the cell type-independent ROSA26 promoter. In the progeny from this cross, Ptfla-driven expression of CRE excises a stop cassette upstream of $l a c Z$, thereby activating the expression of beta-galactosidase. Therefore, the expression of beta-galactosidase marks PTF1A-expressing cells and their progeny [20]. This strategy showed that PTF1A-lineage cells differentiate into duct, acinar and endocrine cells, i.e. all pancreatic epithelial cell types, but not pancreatic mesenchymal cells. We then crossed the Ptfla-Cre:ROSA26-lacZ mice with Ptch $1^{-/ m e s}$ mice to examine whether the increased mesenchymal cell mass expressed beta-galactosidase. Figure 6 shows that the beta-galactosidase-expressing cells in E15.5 pancreas of $P t c h 1^{-/ m e s}$ mice comprised only epithelial cells, as in wild-type mice. Accordingly, lineage-tracing analysis revealed that the extra mesenchymal cells in Ptch1 mutants were not formed via EMT; rather, these cells represented increased mesenchymal cell proliferation. An increased number of BrdU-positive mesenchymal cells in the $\mathrm{Ptcl}^{-/ m e s}$ mice provided further evidence of such proliferative activity (data not shown). Previously, forced expression of Shh under the control of the $P d x 1$ promoter induced transdifferentiation of pancreatic mesenchyme into duodenum mesoderm, which expresses alpha-smooth muscle actin (alpha-SMA) [4]. Immunostaining for alpha-SMA in $P t c 1^{-/ m e s}$ mice pancreas in this study showed staining only in the cells surrounding blood vessels (data not shown). Thus, disruption of Ptch1 in this study did not induce transformation of pancreatic mesenchyme into duodenum mesoderm.

Interestingly, the lineage-tracing analysis of $\mathrm{Ptcl}^{-/ \text {mes }}$ mice revealed beta-galactosidase-expressing epithelial cells in the stomach. This unexpected finding suggests that deficiency in Ptcl function causes a cell fate switch from pancreatic progenitors to stomach epithelial cells.

Decreased beta cell mass in Ptch $1^{-/+}$mice Finally, we investigated whether subtle reductions in Ptc1 function

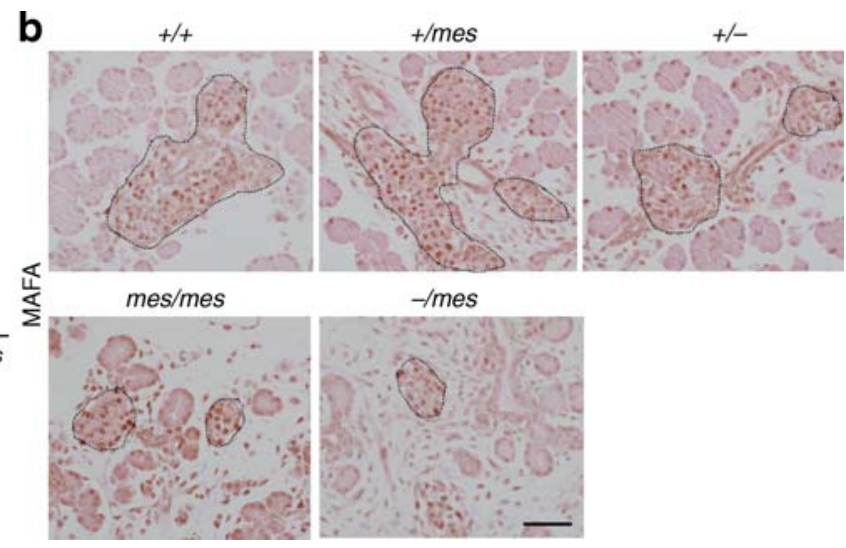

Fig. 5 Expression of key transcription factors for differentiation into pancreatic beta cells in each Ptch1 mutant mice. a Ngn3 mRNA expression levels are shown relative to $\beta$-actin mRNA. To facilitate comparison, expression in wild-type pancreas has been adjusted to 1 . Data are mean \pm SEM. Ptch ${ }^{+/+}$, $n=6 ;$ Ptchl $^{+/ \text {mes }}, n=8 ;$ Ptch $^{+/}$, $n=9 ;$ Ptch $1^{\text {mes } / \text { mes }}, n=6$;

Ptch $1^{-/ m e s}, n=8 ; * p<0.05$ vs Ptchl $^{+/+}$. b MAFA immunostaining of pancreas at E18.5. Scale bar, $50 \mu \mathrm{m}$

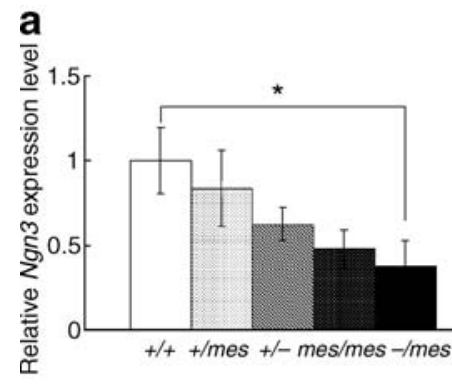



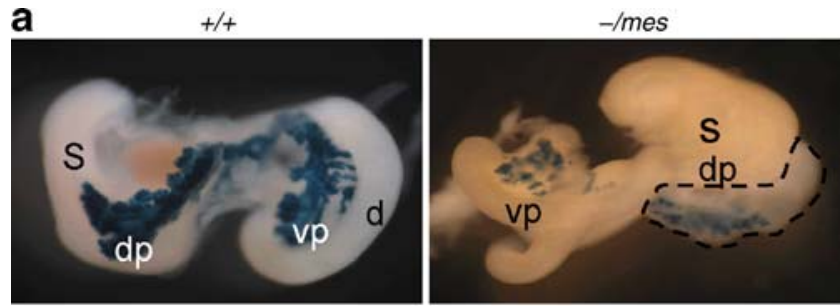

b
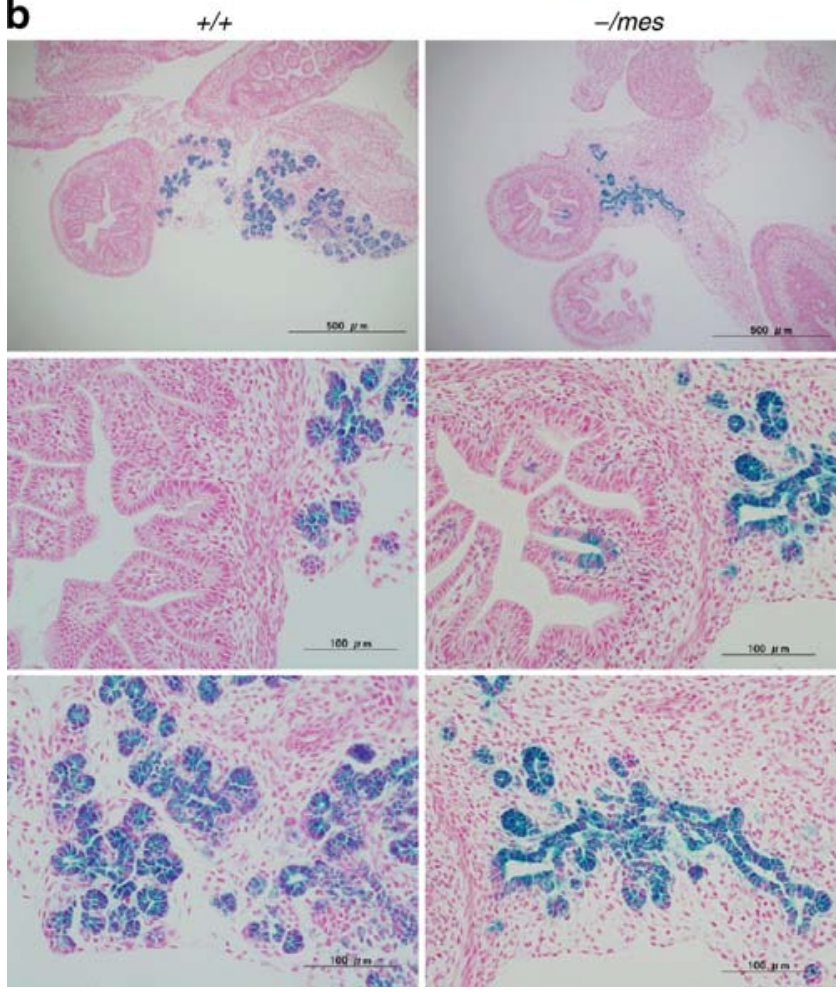

Fig. 6 The origin of increased mesenchyme in $P t c h 1^{-/ m e s}$ mice. a Macroscopic phenotypes of E15.5 pancreas from Ptfla-Cre; ROSA26lacZ mice $(n=7)$ and the trigenic Ptf1a-Cre; Ptch $^{-/ m e s}$; ROSA26-lacZ mice $(n=5)$ stained with X-gal. The black dotted line represents the pancreas. d, duodenum; dp, dorsal pancreas; S, stomach; vp, ventral pancreas. b X-gal staining of pancreatic sections from E15.5 PtflaCre; ROSA26-lacZ mice and the trigenic Ptfla-Cre;Ptch1 ${ }^{-/ m e s}$; ROSA26-lacZ mice. Blue staining for LacZ production marks cells that have undergone CRE-mediated recombination. Scale as indicated

could alter the beta cell mass in adult pancreas, thereby affecting glucose tolerance. For rigorous comparison of glucose tolerance, we used wild-type, $P t c h 1^{+/ m e s}$ and Ptchl $^{+/-}$mice with a C57BL/6 background (backcrossed more than ten generations). At 12 weeks of age, the Ptch $1^{+/ m e s}$ and $P t c h 1^{+/-}$mice showed glucose and insulin levels during IPGTT that were comparable to those of wildtype mice (Fig. 7). Insulin sensitivity of these mice was also similar to wild-types, as assessed by the insulin-tolerance test. However, the area of pancreatic beta cells in $P t c h 1^{+-}$ mice was lower than in wild-type mice. These results confirm that reduced expression of Ptchl can cause decreased beta cell mass in the pancreas. a

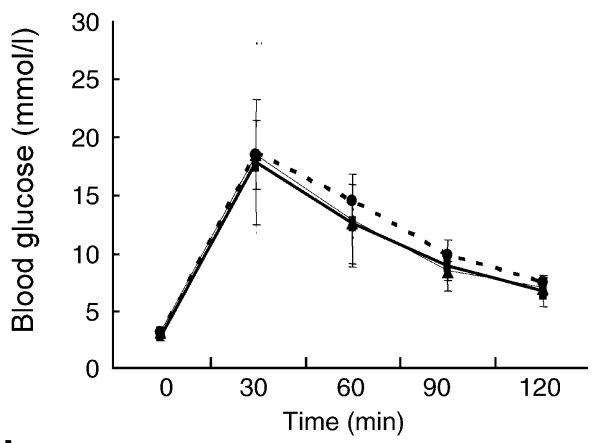

b

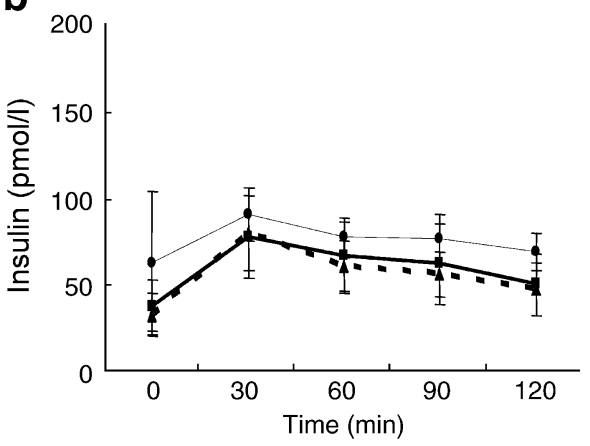

c

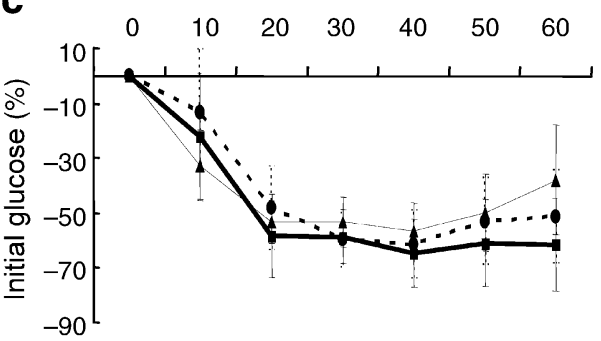

d

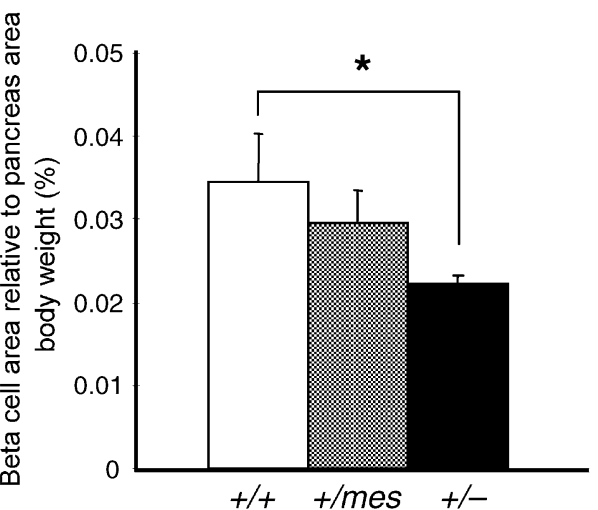

Fig. 7 Glucose tolerance and beta cell mass in Ptch1 mutants. a Blood glucose concentrations after intraperitoneal glucose injection $\left(2 \mathrm{~g} / \mathrm{kg}\right.$ glucose) in 12-week-old, male Ptch $1^{+/ m e s}$ (squares, $n=6$ ), Ptch $^{+/-}$(triangles, $n=4$ ) and wild-type littermates (circles, $n=5$ ). b Serum insulin levels during IPGTT in each group. c Reduction in blood glucose levels after intraperitoneal injection of insulin $(0.75 \mathrm{U} /$ $\mathrm{kg}$ ) in 15 -week-old male Ptch $1^{+/ \text {mes }}$ (squares, $n=13$ ), Ptch $1^{+/-}$ (triangles, $n=12$ ) and wild-type littermates (circles, $n=13$ ). Data are mean \pm SEM. d Beta cell areas in 15 -week-old Ptch $1^{+/ m e s}$ mice $(n=6)$, Ptch $^{+/-}(n=6)$ and wild-type littermates $(n=6)$, shown as a percentage relative to the whole pancreatic area. Data are mean $\pm \mathrm{SEM}$; * $p<0.05$ vs Ptch $1^{+/+}$ 


\section{Discussion}

In this study, we clearly found that mes functions as a hypomorphic allele of Ptch1 in the developing pancreas and that these Ptch 1 mutant mice are useful in ascertaining the dose-dependent effect of increased $\mathrm{HH}$ signalling on pancreatic development.

A previous study suggested that increased $\mathrm{HH}$ signalling inhibits early pancreatic development [4]. It has also been reported that the levels of PDX1 in pancreas at E9.5 in Ptchl $^{-/}$mice was decreased, although Ptchl knockout mice die early during development, making it impossible to perform a detailed analysis of Ptch1 function in them [8]. In this study, the levels of PDX1 and glucagon were not decreased at E10.5 and E12.5 in Ptch1 mutants. Thus, we confirmed that early pancreatic development is disturbed only by total loss of PTCH1 function.

In this study, Ptchl mutants showed a decreased exocrine cell mass in parallel with increased $\mathrm{HH}$ signalling in the pancreas. Similar changes have been reported in PDX1-Shh mice and PAX4-Hh mice [4, 10]. PDX1-Shh mice had only small pancreatic remnants in the duodenal tissue and the phenotype was evidently more severe than in the Ptch1 mutants analysed in this study. PDX1-Shh mice have SHH ectopically expressed very early during pancreatic development. In addition, while PTCH1 was only weakly expressed in pancreatic epithelial cells, the promoter activity of $P d x l$ in pancreas was relatively strong. Thus, $\mathrm{HH}$ signalling in the pancreas of these mice might have exceeded that in our Ptch1 mutant mice. The PAX4-Hh mice in the other study [10] showed a decreased pancreatic cell mass, but the pancreatic tissue was macroscopically visible. The PAX4-Hh phenotype was therefore less severe than in the PDX1-Shh mice and was relatively similar to our $\mathrm{Ptch}^{-/ \text {mes }}$ mice. In addition, HH activity was only upregulated later in development of the PAX4-Hh mice, when expression should be restricted within limited cell lineages such as endocrine cells. While it is possible that increased $\mathrm{HH}$ signalling during early pancreatic development might affect the differentiation of exocrine cells more adversely, the exocrine cell mass in Ptch1 mutant mice decreased in parallel with increasing $\mathrm{HH}$ activation in this study, implying that the phenotype severity might depend on $\mathrm{HH}$ signalling intensity.

All pancreatic endocrine cell types decreased in accordance with the increased $\mathrm{HH}$ signalling in our mutant mice. However, beta cells seemed to be most sensitively affected by the increased $\mathrm{HH}$ activity among the pancreatic epithelial-derived cells. This finding suggests that subtle increases in $\mathrm{HH}$ signalling might induce a decrease in beta cell mass. A decrease in beta cell mass is a typical feature of the onset of type 2 diabetes $[35,36]$. Thus, we investigated the glucose tolerance and beta cell mass in our Ptchl ${ }^{+/ \text {mes }}$ and $P t c h 1^{+/-}$mice after adjusting for genetic background. No differences in glucose tolerance were apparent among wild-type, Ptch $1^{+/ \text {mes }}$ and $P t c h 1^{+/-}$mice. However, a previous study showed that $P t c h 1^{+/}$mice are glucose-intolerant [8]. This discrepancy could be attributed to the different background of mice used. On the other hand, beta cell mass diminished in a stepwise fashion in parallel with the increased $\mathrm{HH}$ signalling, although the difference between wild-type and Ptch $^{+/ m e s}$ mice was not statistically significant. A recent genome-wide association study identified EXT2 as a candidate gene causing type 2 diabetes [37]. The fact that extoses genes modulate $\mathrm{HH}$ signalling [38] implicates these proteins in the development of type 2 diabetes via a decrease in beta cell mass induced by increased HH signalling. Further studies are needed to investigate the relationship of extoses genes and pancreatic beta cell mass.

The Ptch ${ }^{\text {mes/mes }}$ and $\mathrm{Ptch}^{-/ \text {mes }}$ mice in this study showed increased mesenchyme at least in the pancreas, as observed with PAX4-Hh mice [12]. The major phenotypic difference between the mesenchyme of PAX4-Hh mice and $\mathrm{Ptch}^{-/ \text {mes }}$ mice is the presence of alpha-SMA, a marker for duodenal mesenchyme in pancreas. While alpha-SMA was produced in ordinary mesenchymal cells of PAX4-Hh mice, we saw immunostained cells only in the cells surrounding blood vessels within the pancreas [12]. In this regard, the mesenchyme phenotype of Ptch $^{- \text {mes }}$ mice might be more similar to the previously described $\mathrm{Hhip}^{-/} ; \mathrm{Ptchl}^{+/-}$mice, which showed a subtle increase in mesenchymal cells and no presence of alpha-SMA in mesenchyme [10].

EMT is a process by which epithelial cells lose their mature characteristics and acquire properties associated with mesenchymal cells. While two studies have suggested that under certain conditions pancreatic beta cells can undergo EMT into fibroblast-like cells under culture [32, 33], a recent study used lineage tracing to demonstrate that islet-derived fibroblast-like cells were not EMT-derived [34]. The increased pancreatic mesenchyme observed in our Ptch1 mutants resembled an EMT event. However, lineagetracing analysis also revealed here that the increased mesenchyme in the Ptch1 $1^{- \text {mes }}$ mice was not derived via EMT. BrdU staining confirmed that the increased mesenchymal mass reflected proliferation of pancreatic mesenchymal cells. Interestingly, the lineage tracing also revealed a cell fate switch from pancreatic epithelial cell progenitors to stomach epithelial cells in the Ptch1 $1^{-/ m e s}$ mice. This cell fate change warrants further detailed characterisation.

In conclusion, our study demonstrated that dysfunctional PTCH1 decreases pancreatic endocrine and exocrine cell mass in a dose-dependent manner, with the beta cell mass particularly responsive to subtle reductions in PTCH1 function and expression. Decreased beta cell mass is a common pathophysiological feature of type 2 diabetes. 
Together with data on EXT2 as a candidate gene causing type 2 diabetes, our data functionally link $\mathrm{HH}$ signalling with the development of the disease.

Acknowledgements We thank N. Daimaru and E. Magoshi for their excellent technical assistance, J. Motoyama for Ptch1 mutant mice, P. Soriano for ROSA26-lacZ mice and C.V.E. Wright for Ptfla-Cre mice. This work was supported by grants from the Ministry of Education, Sports and Culture of Japan (to H. Watada).

Duality of interest The authors declare that there is no duality of interest associated with this manuscript.

\section{References}

1. Ingham PW, McMahon AP (2001) Hedgehog signaling in animal development: paradigms and principles. Genes Dev 15:30593087

2. Nieuwenhuis E, Motoyama J, Barnfield PC et al (2006) Mice with a targeted mutation of patched 2 are viable but develop alopecia and epidermal hyperplasia. Mol Cell Biol 26:6609-6622

3. Nieuwenhuis E, Hui CC (2005) Hedgehog signaling and congenital malformations. Clin Genet 67:193-208

4. Apelqvist A, Ahlgren U, Edlund H (1997) Sonic hedgehog directs specialised mesoderm differentiation in the intestine and pancreas. Curr Biol 7:801-804

5. Hebrok M, Kim SK, Melton DA (1998) Notochord repression of endodermal Sonic hedgehog permits pancreas development. Genes Dev 12:1705-1713

6. Kim SK, Melton DA (1998) Pancreas development is promoted by cyclopamine, a hedgehog signaling inhibitor. Proc Natl Acad Sci U S A 95:13036-13041

7. Lau J, Kawahira H, Hebrok M (2006) Hedgehog signaling in pancreas development and disease. Cell Mol Life Sci 63:642-652

8. Hebrok M, Kim SK, St Jacques B, McMahon AP, Melton DA (2000) Regulation of pancreas development by hedgehog signaling. Development 127:4905-4913

9. Thomas MK, Rastalsky N, Lee JH, Habener JF (2000) Hedgehog signaling regulation of insulin production by pancreatic beta-cells. Diabetes 49:2039-2047

10. Kawahira H, Ma NH, Tzanakakis ES, McMahon AP, Chuang PT, Hebrok M (2003) Combined activities of hedgehog signaling inhibitors regulate pancreas development. Development $130: 4871-4879$

11. Hebrok M (2003) Hedgehog signaling in pancreas development. Mech Dev 120:45-57

12. Kawahira H, Scheel DW, Smith SB, German MS, Hebrok M (2005) Hedgehog signaling regulates expansion of pancreatic epithelial cells. Dev Biol 280:111-121

13. Johnson RL, Milenkovic L, Scott MP (2000) In vivo functions of the patched protein: requirement of the $\mathrm{C}$ terminus for target gene inactivation but not Hedgehog sequestration. Mol Cell 6:467-478

14. Lu X, Liu S, Kornberg TB (2006) The C-terminal tail of the Hedgehog receptor Patched regulates both localization and turnover. Genes Dev 20:2539-2551

15. Makino S, Masuya H, Ishijima J, Yada Y, Shiroishi T (2001) A spontaneous mouse mutation, mesenchymal dysplasia (mes), is caused by a deletion of the most C-terminal cytoplasmic domain of patched (ptc). Dev Biol 239:95-106

16. Jessell TM (2000) Neuronal specification in the spinal cord: inductive signals and transcriptional codes. Nat Rev Genet 1:20-29

17. Nieuwenhuis E, Barnfield PC, Makino S, Hui CC (2007) Epidermal hyperplasia and expansion of the interfollicular stem cell compartment in mutant mice with a C-terminal truncation of Patched1. Dev Biol 308:547-560

18. Goodrich LV, Milenkovic L, Higgins KM, Scott MP (1997) Altered neural cell fates and medulloblastoma in mouse patched mutants. Science 277:1109-1113

19. Soriano P (1999) Generalized lacZ expression with the ROSA26 Cre reporter strain. Nat Genet 21:70-71

20. Kawaguchi Y, Cooper B, Gannon M, Ray M, MacDonald RJ, Wright CV (2002) The role of the transcriptional regulator Ptfl a in converting intestinal to pancreatic progenitors. Nat Genet 32:128-134

21. Livak KJ, Schmittgen TD (2001) Analysis of relative gene expression data using real-time quantitative PCR and the 2(-Delta Delta C(T)) Method. Methods 25:402-408

22. Iwashita N, Uchida T, Choi JB et al (2007) Impaired insulin secretion in vivo but enhanced insulin secretion from isolated islets in pancreatic beta cell-specific vascular endothelial growth factor-A knock-out mice. Diabetologia 50:380-389

23. Watada H, Kajimoto Y, Umayahara Y et al (1996) The human glucokinase gene beta-cell-type promoter: an essential role of insulin promoter factor 1/PDX-1 in its activation in HIT-T15 cells. Diabetes 45:1478-1488

24. Choi JB, Uchino H, Azuma K et al (2003) Little evidence of transdifferentiation of bone marrow-derived cells into pancreatic beta cells. Diabetologia 46:1366-1374

25. Fujitani Y, Fujitani S, Boyer DF et al (2006) Targeted deletion of a cis-regulatory region reveals differential gene dosage requirements for Pdx 1 in foregut organ differentiation and pancreas formation. Genes Dev 20:253-266

26. Uchida T, Iwashita N, Ohara-Imaizumi M et al (2007) Protein kinase Cdelta plays a non-redundant role in insulin secretion in pancreatic beta cells. J Biol Chem 282:2707-2716

27. Edlund H (1998) Transcribing pancreas. Diabetes 47:1817-1823

28. Apelqvist A, Li H, Sommer L et al (1999) Notch signalling controls pancreatic cell differentiation. Nature 400:877-881

29. Schwitzgebel VM, Scheel DW, Conners JR et al (2000) Expression of neurogenin3 reveals an islet cell precursor population in the pancreas. Development 127:3533-3542

30. Matsuoka TA, Artner I, Henderson E, Means A, Sander M, Stein R (2004) The MafA transcription factor appears to be responsible for tissue-specific expression of insulin. Proc Natl Acad Sci U S A 101:2930-2933

31. Bhushan A, Itoh N, Kato $\mathrm{S}$ et al (2001) Fgfl0 is essential for maintaining the proliferative capacity of epithelial progenitor cells during early pancreatic organogenesis. Development 128:5109-5117

32. Gershengorn MC, Hardikar AA, Wei C, Geras-Raaka E, MarcusSamuels B, Raaka BM (2004) Epithelial-to-mesenchymal transition generates proliferative human islet precursor cells. Science 306:2261-2264

33. Ouziel-Yahalom L, Zalzman M, Anker-Kitai L et al (2006) Expansion and redifferentiation of adult human pancreatic islet cells. Biochem Biophys Res Commun 341:291-298

34. Chase LG, Ulloa-Montoya F, Kidder BL, Verfaillie CM (2007) Islet-derived fibroblast-like cells are not derived via epithelialmesenchymal transition from $\mathrm{Pdx}-1$ or insulin-positive cells. Diabetes 56:3-7

35. Butler AE, Janson J, Bonner-Weir S, Ritzel R, Rizza RA, Butler PC (2003) Beta-cell deficit and increased beta-cell apoptosis in humans with type 2 diabetes. Diabetes 52:102-110

36. Rhodes CJ (2005) Type 2 diabetes-a matter of beta-cell life and death? Science 307:380-384

37. Sladek R, Rocheleau G, Rung J et al (2007) A genome-wide association study identifies novel risk loci for type 2 diabetes. Nature 445:881-885

38. Bellaiche Y, The I, Perrimon N (1998) Tout-velu is a Drosophila homologue of the putative tumour suppressor EXT-1 and is needed for Hh diffusion. Nature 394:85-88 